\title{
The research on lapatinib in autophagy, cell cycle arrest and epithelial to mesenchymal transition via Wnt/ErK/PI3K-AKT signaling pathway in human cutaneous squamous cell carcinoma
}

Ming Yao ${ }^{1,2^{*}}$, Yuan-Yuan Shang ${ }^{2,3^{*}}$, Zhi-Wei Zhou' ${ }^{2}$, Yin-Xue Yang ${ }^{4}$, Yin-Sheng Wu ${ }^{1}$, Li-Feng Guan ${ }^{1}$, Xin-Yu Wang 2,5 , Shu-Feng Zhou ${ }^{2 \bowtie}, X_{i} W^{6 \bowtie}$

1. Department of Burns and Plastic Surgery, General Hospital, Ningxia Medical University, Yinchuan, People's Republic of China.

2. Department of Pharmaceutical Sciences, College of Pharmacy, University of South Florida, Tampa, FL, USA.

3. Department of Dermatology, General Hospital, Ningxia Medical University;

4. Department of Colorectal Surgery, General Hospital, Ningxia Medical University;

5. Department of Pharmacy, General Hospital, Ningxia Medical University, Yinchuan;

6. Department of Diagnostic and Therapeutic Ultrasonography, Tianjin Medical University Cancer Institute and Hospital, National Clinical Research Center of Cancer, Key Laboratory of Cancer Prevention and Therapy, Tianjin, China.

* These authors Ming Yao and Yuan-Yuan Shang contributed equally to this work.

$\triangle$ Corresponding authors: Prof. Shu-Feng Zhou, Department of Pharmaceutical Sciences, College of Pharmacy, University of South Florida, 12901 Bruce B Downs Blvd, MDC30, Tampa, FL 33612, USA. Email: szhou@health.usf.edu. Prof. Xi Wei, MD, PhD, Department of Diagnostic and Therapeutic Ultrasonography, Tianjin Medical University Cancer Institute and Hospital, National Clinical Research Center of Cancer, Key Laboratory of Cancer Prevention and Therapy, Tianjin, China, weixi@tmu.edu.cn.

(C) Ivyspring International Publisher. This is an open access article distributed under the terms of the Creative Commons Attribution (CC BY-NC) license (https://creativecommons.org/licenses/by-nc/4.0/). See http://ivyspring.com/terms for full terms and conditions.

Received: 2016.07.14; Accepted: 2016.11.10; Published: 2017.01.15

\begin{abstract}
Cutaneous squamous cell carcinoma (cSCC) contributes to one of most common types of skin cancer. Epidermal growth factor receptor (EGFR) activation has been investigated to be associated with the development of cSCC. Lapatinib is an inhibitor targeting HER2/neu and EGFR pathway. We found that lapatinib can inhibit proliferation by enhancing apoptosis of human CSCC cell lines. The cSCC cell cycle distribution could be arrested in G2/M phase after lapatinib treatment. In the in vitro experiment, we found that lapatinib interrupted PI3K/AKT/mTOR signaling pathway in human cSCC cells. Furthermore, lapatinib could suppress epithelial to mesenchymal transition (EMT) via $\mathrm{Wnt} /$ ErK/PI3K-AKT signaling pathway to represent a promising anticancer drug for cSCC treatment.
\end{abstract}

Key words: Lapatinib, cutaneous squamous cell carcinoma (cSCC), proliferation, epithelial to mesenchymal transition.

\section{Introduction}

Cutaneous squamous cell carcinoma (cSCC) has been seen as the second most common type of skin cancer, defined as an uncontrolled growth of abnormal cells arising from the squamous cells[1]. Prolonged exposure to ultraviolet (UV) radiation and Chemical carcinogens increase the risk of squamous cell carcinoma[2]. Cutaneous squamous cell carcinoma is not life-threatening, but if untreated, it can grow large or to spread to other organs, causing serious complications, local lymph nodes involvement, and distant metastases. In 2012, an estimated 700,000 cases of CSCC were diagnosed and 3,900 to 8,800 patients died each year in the US[3]. So far, squamous cell carcinoma is detected at an early stage and surgical excision is almost always curable to cause minimal damage.

Previous studies reported that nonaspirin nonsteroidal anti-inflammatory drugs (NSAIDs) significantly reduced the risk of cSCC development [4]. Aspirin usage reduced the risk of cSCC by $12 \%$, which was of borderline significance [5]. However, it is still necessary to seek out an efficacy treatment 
strategy for cSCC. Lapatinib is a dual tyrosine kinase inhibitor which blocks the HER2/neu and epidermal growth factor receptor (EGFR) pathways [6], which is an orally active drug for breast cancer and other solid tumors [7]. As we known, growth factors and receptors play essential roles in the regulation of epithelial cell proliferation, and abnormalities in their expression and signaling pathways, contributing to progression and maintenance of the malignant phenotype in human cancers[8]. The deregulation of EGFR activation has been shown to be closely associated with the development and progression of cSCC[9]. Christopher J. et al. [10] reported that the EGFR inhibitor, interrupted EGF-induced cytoskeleton remodeling, cell growth and invasive phenotype of a cutaneous squamous cell. However, cSCC is the most aggressive cancer with very poor prognosis. The optimal treatment of cSCC remains a major challenge. So far, effects of lapatinib to treat cSCC and its underlying mechanism have not been illustrated. Thus, in this study, we explored lapatinib inhibition of skin squamous cell and fibroblasts cells via epithelial to mesenchymal transition (EMT) signaling pathway.

\section{Materials and methods}

\section{Cell lines and cell culture}

Human squamous cell carcinoma (cSCC) cells were obtained from the American Type Culture Collection (Manassas, VA, USA). Cell lines were maintained in Dulbecco's Modified Eagle's Medium supplemented with $10 \%$ heat-inactivated fetal bovine serum and $1 \%$ penicillin in a $5 \% \mathrm{CO} 2$ incubator at $37^{\circ} \mathrm{C}$. Lapatinib was dissolved in dimethyl sulfoxide at a stock concentration of $50 \mathrm{mM}$ and stored at $-20^{\circ} \mathrm{C}$.

\section{Chemicals and reagents}

Cell lines were maintained in DMEM medium supplemented with 10\% FCS were sourced from Corning Cellgro Inc (Herndon, VA, USA). A Cyto-ID autophagy detection kit was sourced from Enzo Life Sciences Inc (Farmingdale, NY, USA). 4, 6-Diamidino-2-phenylindole (DAPI) was obtained from Invitrogen (Carlsbad, CA, USA). The thiazolyl blue tetrazolium bromide (MTT) was purchased from Sigma-Aldrich Inc (St Louis, MO, USA). An Annexin $\mathrm{V}$ : phycoerythrin (PE) apoptosis detection kit was obtained from BD Biosciences Inc (San Jose, CA, USA). Primary antibodies against human were all purchased from Cell Signaling Technology Inc (Beverly, MA, USA). The antibody against human $\beta$-actin was purchased from Santa Cruz Biotechnology Inc (Santa Cruz, CA, USA).

\section{Cell viability assay}

The effect of lapatinib on the viability of cSCC A431 cells was examined using the MTT assay as previous described. Briefly, A431 cells were seeded into a 96-well plate at a density of 8,000 cells/well. After the incubation for 24 hours, A431 cells were treated with lapatinib at concentrations ranging from 0.1 to $10 \mu \mathrm{M}$ for 24 and 48 hours with a volume of $100 \mu \mathrm{L}$ medium. And then they were detected by the general procedure for MTT at a wavelength of 450 $\mathrm{nm}$. The concentration required for $50 \%$ inhibition of growth (IC50) was analyzed using GraphPad Prism 6 software (San Diego, CA, USA).

\section{Analysis of cell cycle distribution}

The effect of lapatinib on the cell cycle distribution of A431 cells was examined using flow cytometry. Briefly, two cell lines were treated with lapatinib at concentrations of $0.1,1$, and $5 \mu \mathrm{M}$ for 48 hours. In separate experiments, they were treated with $5 \mu \mathrm{M}$ lapatinib for $0,24,48$ and 72 hours. After dealing with the cell cycle routine process, a total number of $1 \sim 2 \times 10^{4}$ cells were subject to cell cycle analysis using a flow cytometer (Becton Dickinson Immunocytometry Systems, San Jose, CA, USA). The positive gate was set according to previous studies $[11,12]$.

\section{Quantification of cellular apoptosis}

The effect of lapatinib on apoptosis of A431 cells was quantitated using the Annexin V: PE apoptosis detection kit according to the manufacturer's instructions. Cells were treated with lapatinib at concentrations of $0.1,1$ and $5 \mu \mathrm{M}$ for 48 hours and then dealt with the protocol of cellular apoptosis. The apoptosis analyzes by a flow cytometer (Becton Dickinson Immunocytometry Systems, San Jose, CA, USA).

\section{Determination of cellular autophagy}

To determine the effect of lapatinib on autophagy in A431 cells, the intracellular autophagy level was examined using flow cytometry. The A431 cells were treated with concentration of lapatinib at $0.1,1$ and $5 \mu \mathrm{M}$ for 48 hours. After 48 hours of incubation, the cells were detected with the protocol of cellular autophagy. And then autophagy was determined using the green (FL1) channel of a flow cytometer.

\section{Confocal fluorescence microscopy}

Confocal microscopic analysis was performed to further examine the lapatinib-induced autophagy in A431 cells using a Cyto-ID autophagy detection kit. After testing the process, it was examined using a TCS 
SP2 laser scanning confocal microscope (Leica, Wetzlar, Germany) by a standard fluorescein isothiocyanate filter set for imaging the autophagy signal at wavelengths of $405 / 488 \mathrm{~nm}$.

\section{Western blot analysis}

The levels of various cellular proteins related to the cell cycle, apoptosis, EMT and autophagy were determined using Western blotting assays. A431 cell lines were washed with phosphate-buffered saline after 48 hours of treatment with lapatinib at $0.1,1$ and $5 \mu \mathrm{M}$. The concentration of protein was collected by the routine process, using the Pierce bicinchoninic acid protein assay kit. The available protein with thermal denaturation at $100^{\circ} \mathrm{C}$ was for 5 minutes. The appropriate steps were followed as previous studies. Visualization was performed using an enhanced chemiluminescence kit (BioRad Inc, Hercules, CA, USA). The date was analyzed using Image Lab 3.0 (BioRad Inc). The protein level was normalized to match densitometry value of the internal control.

\section{Statistical analysis}

All of the data statistics were analyzed by the GraphPad Prism 6 software (San Diego, CA, USA). The data were presented as the mean \pm standard deviation. Comparisons of multiple groups were achieved by one-way analysis of variance following by Tukey's multiple comparison procedures. $P<0.05$ were considered to be statistical differences significantly. All of the assays were performed at least three times independently.

\section{Results}

\section{Lapatinib inhibited A431 cell lines' proliferation in a time-dependent and dose-dependent manner}

After culturing with different concentrations of lapatinib (Figure 1A) for 24 and 48-hour, the inhibitory effect of lapatinib on the proliferation of A431 cells was further confirmed by an MTT assay. After stimulating with different concentrations of lapatinib for 24 and 48-hour, the dramatical decrease

A

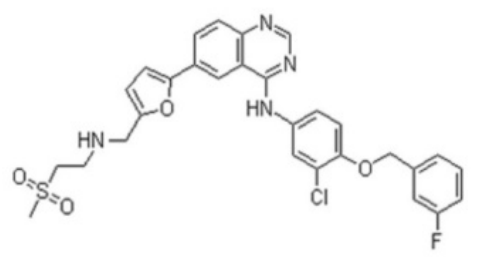

of A431 cells growth was observed compared with the control groups. The peak was reached on stimulation with $50 \mu \mathrm{mol} / 1$ lapatinib for $48 \mathrm{~h}$ (Figure 1B). These results indicated that lapatinib significantly inhibited the proliferation of A431 cells in a time-dependent and dose-dependent manner. The $\mathrm{IC}_{50}$ values were $4.46 \mu \mathrm{M}$ and $15.46 \mu \mathrm{M}$ for A431 cell line after 24 and 48-hour incubation with lapatinib, respectively (Figure 1B). The results indicated that lapatinib exerts a potent inhibitory effect on cell proliferation in A431 cell line.

\section{Lapatinib promoted A431 cell lines' apoptosis by suppressing FADD, caspase $8, \mathrm{Bcl}-2$, and MEK}

The apoptosis of A431 cells was measured by FCM after pretreating with $5 \mu \mathrm{M}$ lapatinib for 48 hours. Results showed that in parallel with the control groups, there was a significant increase in apoptosis of A431 cells (Figure 2 A and B). To provide much evidence to this potential phenotype in A431 cell lines, we detected FADD, Bcl-2, caspase 8, ERK and MEK markers with increasing dose of lapatinib in western blot assay, indicating lapatinib has ability to decrease P-FADD, caspase 8, Bcl-2, P-ERK and P-MEK expression but without induction of FADD level (Figure 2C).

\section{Lapatinib regulated the cell cycle distribution of A431 cells}

We examined the effect of lapatinib on cell cycle distribution in A431 cells using flow cytometry. When being treated with lapatinib at $0.1,1$ and $5 \mu \mathrm{M}$ for 48 hours, respectively, lapatinib significantly induced G2/M phase arrest in A431 cell lines (Figure 3 A-C). In separate experiments, we examined the effect of lapatinib on cell cycle distribution during 72 hours. In comparison to control cells, the percentage of A431 cells in G2/M phase increased from 4 to 72-hour with treatment of $5 \mu \mathrm{M}$ lapatinib (Figure $4 \mathrm{~A}-\mathrm{C}$ ). The data indicated that lapatinib treatment remarkably induces A431 cell line arrest in G2/M phase, decreasing proliferation of cSCC cells in vitro.

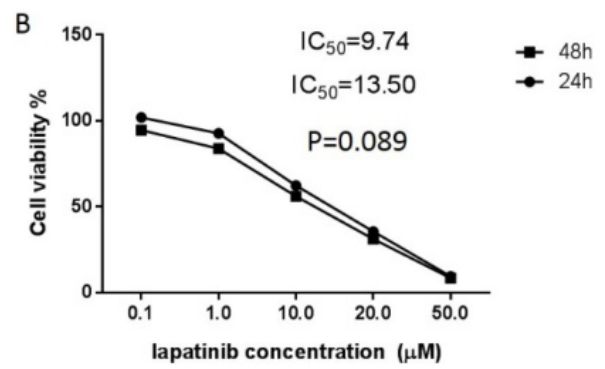

Figure 1. Lapatinib as an inhibitor contributes to gastric cancer cells suppression in vitro. A. Chemical structure of lapatinib; B. The MTT assay was performed on the viability of $A 431$ cells by lapatinib at different concentrations $(0.1-50 \mu \mathrm{M})$ and $I_{50}$ value was analyzed as indicated on the plot (t-test). 


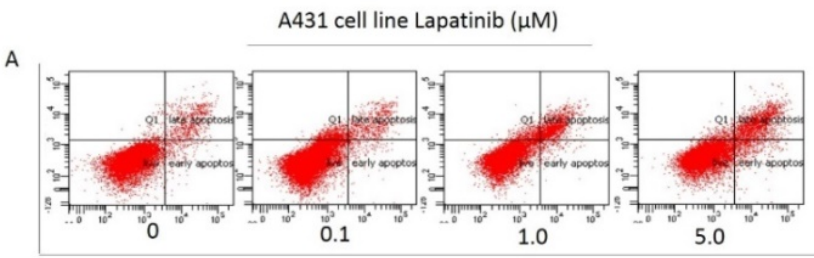

B

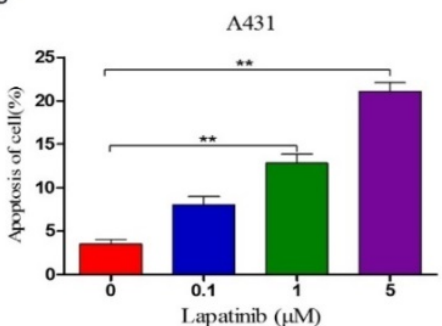

C A431 cell line Lapatinib $(\mu \mathrm{M})$

$\begin{array}{llll}0 & 0.1 & 1.0 & 5.0\end{array}$

**, $P<0.05)$; C. The protein markers as shown on figure were detected by western blot assay.

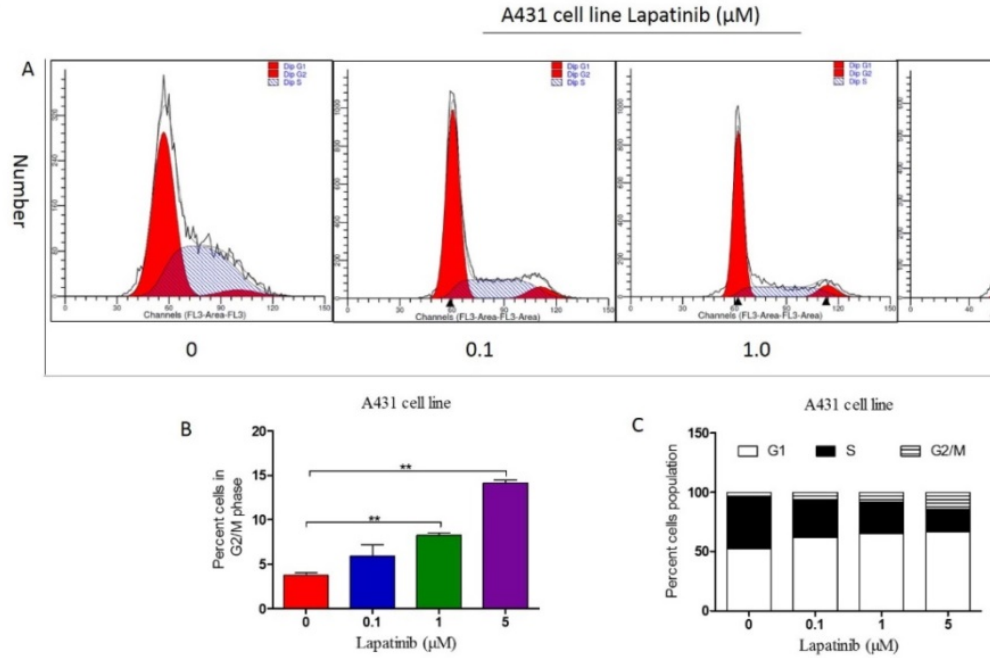

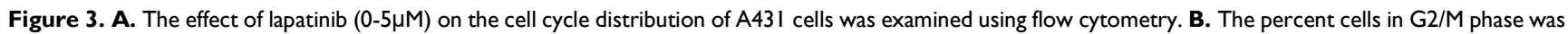
analyzed on the graph (t-test; $\left.{ }^{* *}, P<0.05\right)$; C. The percent cells population in cell distribution was calculated on the plot.
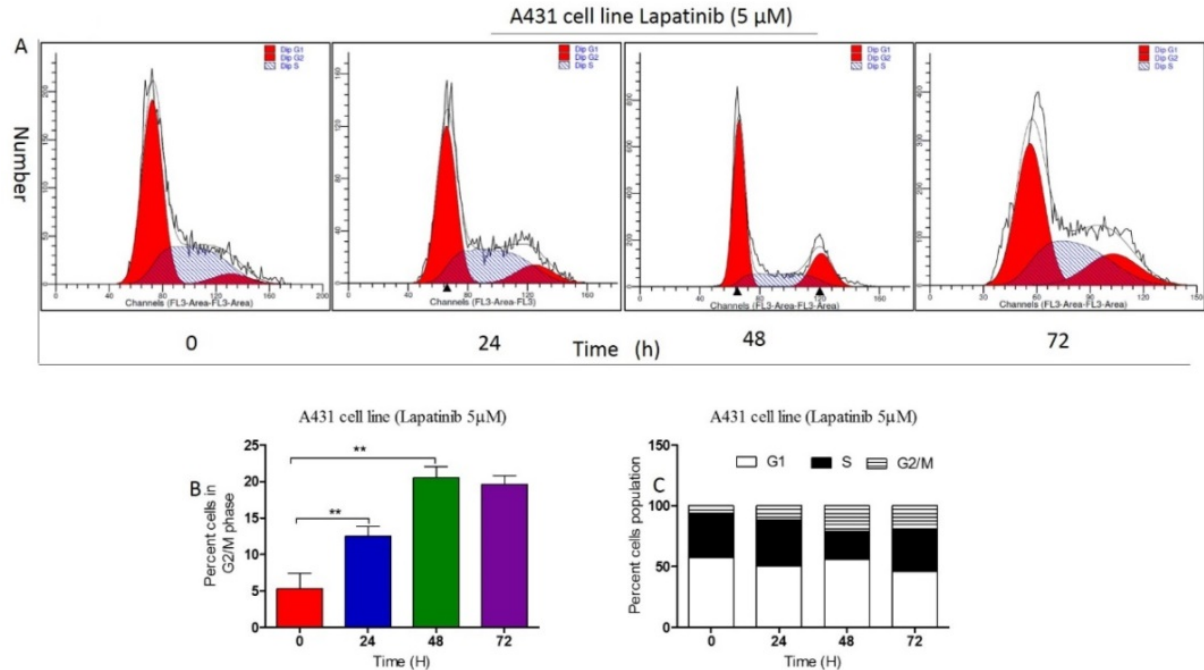

Figure 4. A. The fixed concentration of lapatinib $(5 \mu \mathrm{M})$ on the cell cycle distribution of $\mathrm{A} 43 \mathrm{I}$ cells was examined using flow cytometry. B. The percent cells in $\mathrm{G} 2 / \mathrm{M}$ phase was analyzed on the graph (t-test; **, $\mathrm{P}<0.05)$; $\mathbf{C}$. The percent cells population in cell distribution was calculated on the plot. 


\section{Lapatinib induced autophagy in A431 cell line by suppressing PI3K/AKT/mTOR signaling pathway in vitro}

In order to illustrate the role of lapatinib in autophagy of cutaneous squamous cell carcinoma cells, we used Cyto-IDr fluorescent probe autophagy detection assay to exam autophagy cell markers in A431 cell line. The results showed that after treatment of A431 cells by different concentration of lapatinib $(0.1 \mu \mathrm{M}, 1 \mu \mathrm{M}$ and $5 \mu \mathrm{M})$, green autophagy dye was shown to gather around cells (Figure $5 \mathrm{~A}$ and $\mathrm{B}$ ), and indicating lapatinib was able to induce autophagy in cutaneous squamous cell carcinoma. Moreover, the number of autophagic cells increased according to dose-dependent lapatinib treatment using flow cytometry assay (Figure $6 \mathrm{~A}$ and B).

When analyzed by western blot, our data showed lapatinib treatment $(0.1,1$ and $5 \mu \mathrm{M})$ caused significant alterations in the expression and phosphorylation levels of key functional proteins which regulating autophagy signaling pathway. There was decreasing phosphorylation levels of p-Akt, and p-mTOR and increasing level of p-PTEN in A431 cell line as Figure 6C shown.

\section{Lapatinib inhibited EMT phenotype in A431 cell line via WNT/ $\beta$-catenin/ E-cadherin pathway}

Epithelial to mesenchymal transition (EMT) is a critical process involved in cancer invasion and metastasis, with the decreased expression of cell adhesion molecules among cells[11, 13]. The previous data demonstrated that EMT-related proteins expression was associated with enhanced malignancy in a variety of tumors [11, 13-15]. When we analyzed the expression of E-cadherin and Vimentin in A431 cell treated with lapatinib $(0.1,1$ and $5 \mu \mathrm{M})$ for 48 hours by immunofluorescence, the results also showed that lapatinib can induce the E-cadherin but inhibit Wnt, $\beta$-catenin, Slug, N-cadherin and
Vimentin expression by western blot (Figure 7A and B). Taken together, we conclude that lapatinib suppresses the EMT probably through $\mathrm{WNT} / \beta$-catenin/E-cadherin pathway in cutaneous squamous cell carcinoma cells.

\section{Discussion}

Cutaneous squamous cell carcinoma (cSCC) has been considered as one of most common type of non-melanoma skin cancer with increasing incidence worldwide [16, 17]. Although local surgery contributes to its main treatment, there is still an urgent need for new therapeutic strategies for cSCC. Lapatinib is reported to involve in epidermal growth factor receptor (EGFR) pathways. Some previously studies have reported squamous cell carcinoma can express epidermal growth factor receptor_EGFR) protein [18]. However, to our knowledge, there are few studies about lapatinib in treatment of cSCC, while some studies focus on combination of lapatinib and trastuzumab in treatment of Her-2 positive breast cancer[19]. A phase II study for Lapatinib plus capecitabine was presented as the first-line treatment for patients with advanced or metastatic gastric cancer. In this case, we further investigate the role of lapatinib in treatment of human CSCC in vitro and in vivo to illustrate the mechanism of this small molecular targeted drug.

To investigate how lapatinib treats the CSCC by inhibiting EGFR, we explored the anticancer effect of lapatinib on the human CSCC cell line. In the in vitro experiment, we found that lapatinib prevents the proliferation of A431 cell line effectively in a time-dependent and dose-dependent manner, which is consistent with Liu et al.[20] report. We further analyzed the apoptosis of cSCC cells induced by lapatinib. The assays showed that lapatinib enhanced apoptosis in A431 cell line cells through FADD, Bcl-2, and P-MEK/ERK signaling pathway, which suggests a potential anticancer activity of lapatinib.
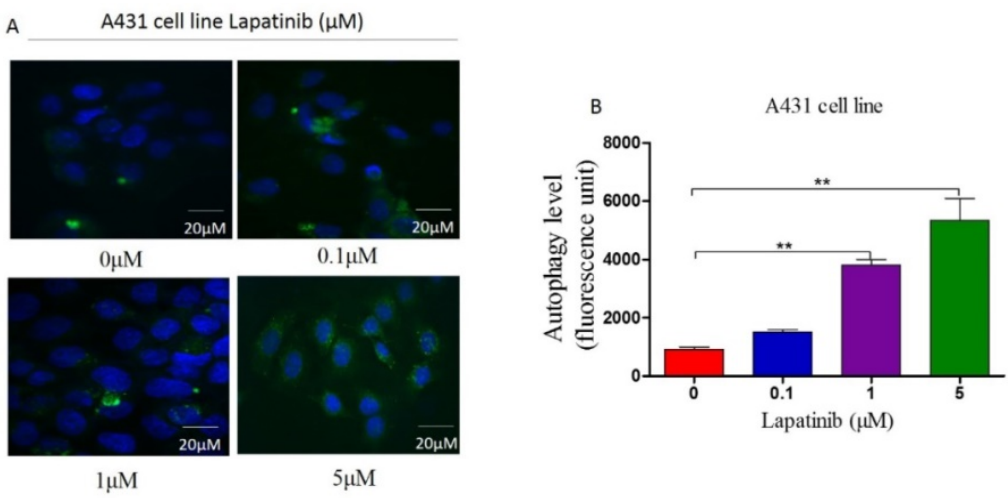

Figure 5. A. The cellular autophagy induced by the concentration of lapatinib (0-5 $\mu \mathrm{M})$ by confocal microscopy with the application of Cyto-ID autophagy detection kit. Immunofluorescence. (Magnification: 1000x). B. A number of autophagy A431 cells was analyzed on the graph. 

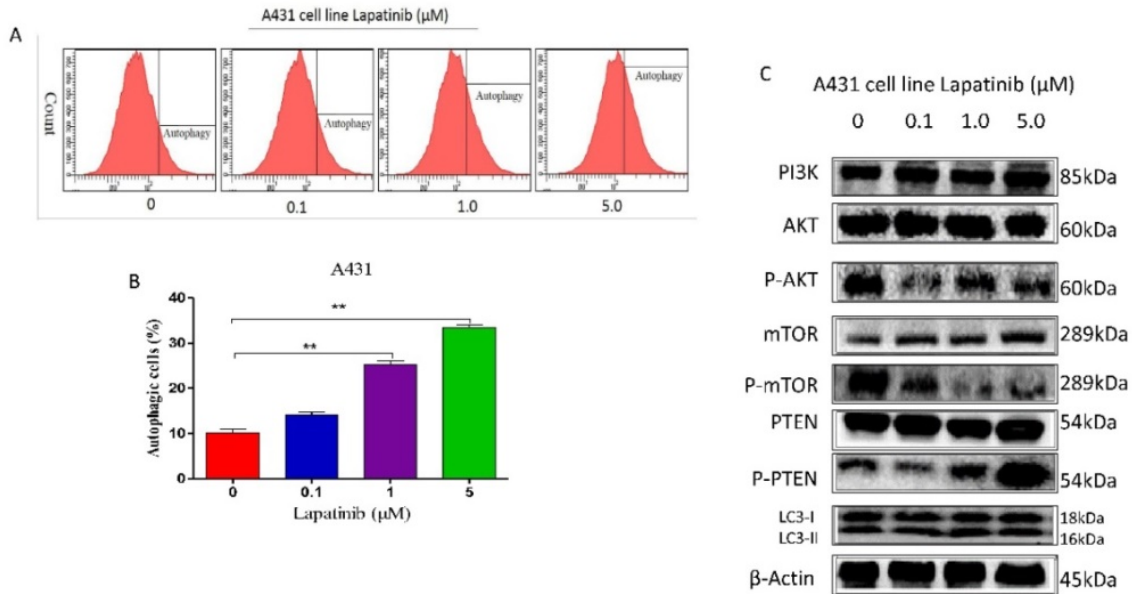

Figure 6. A. The cellular autophagy induced by the concentration of lapatinib $(0-5 \mu \mathrm{M})$ by confocal microscopy with the application of Cyto-ID autophagy detection kit. B. The percentage of autophagic A43I cells was stained by the Cyto-ID autophagy detection kit and analyzed using the green (FLI) channel of the flow cytometer. C. The protein markers as shown on figure were detected by western blot assay.
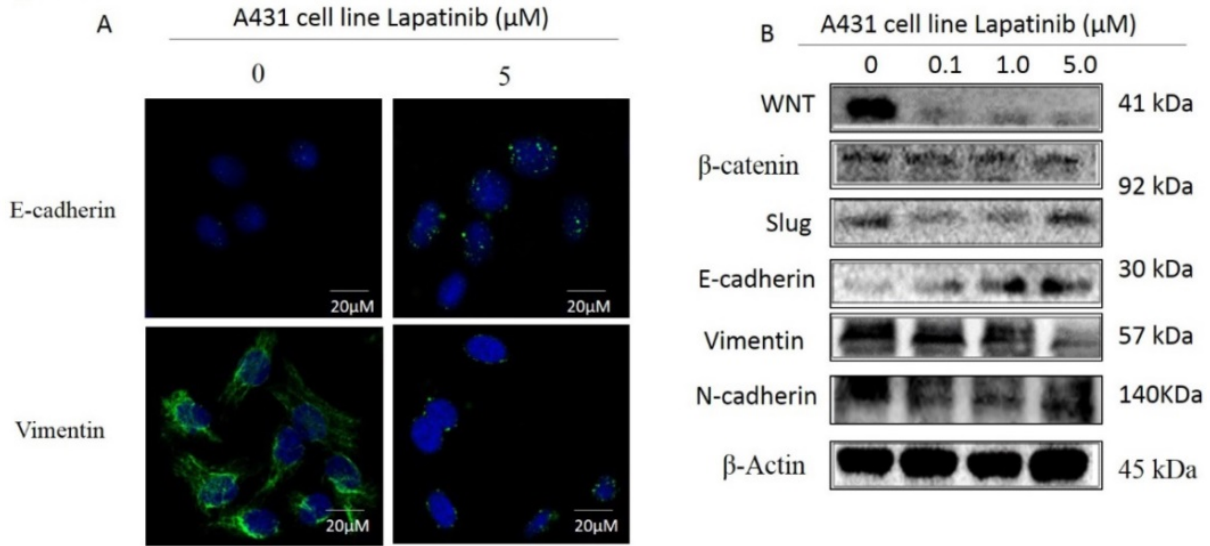

Figure 7. A. Cells treated with lapatinib $(5 \mu \mathrm{M})$ increased the expression of E-cadherin and inhibited the expression of Vimentin by immunofluorescence.

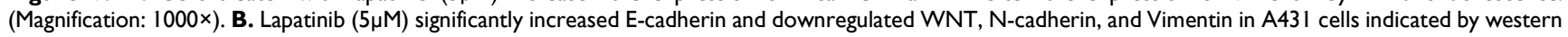
blot.

The biomarkers including AKT, mTOR and PTEN exerts an important role in the regulation of chromosome assembly and its inactivation of these proteins results in G2/M arrest. In the present study, our findings clearly showed that lapatinib treatment remarkably decreased the ratio of p-AKT and p-mTOR over AKT and mTOR in A431 cell lines, suggesting that lapatinib may induce cell cycle arrest in G2/M phase. Our findings also showed that lapatinib exhibits potent inhibitory effects on cSCC cell growth and cell cycle progression. Lapatinib exerts a potent $\mathrm{CSCC}$ cell-killing effect via autophagic cell death with the involvement of PI3K/Akt/mTOR.

Furthermore, our data indicated that lapatinib treatment can significantly increase the expression levels of WNT/ $\beta$-cadherin pathway, which serves as an inhibitor of cell cycle progression via inhibition of CDC2 activity. Therefore, increased expression of $\mathrm{WNT} / \beta$-cadherin contributes to cell cycle arrest in A431 cell line. Numerous evidence has shown that aberrant Wnt/ $\beta$ - catenin signaling accounts for tumor initiation and inhibits mitochondrial apoptosis. In addition, the treating A431 cell with lapatinib results in suppression of Wnt/ $\beta$-catenin activation, which has been shown to inhibit the proliferation, tumorigenesis, and metastasis of various cancer cells.

Lapatinib represented a substantial suppressing effect on EMT in cSCC cells. There is a negative regulation loop between E-cadherin-mediated calcium-dependent cell-cell adhesion and the snail/slug[21]. Inhibition of snail/slug expression enable to suppress the EMT progression in CSCC. Our findings showed that lapatinib treatment significantly increases the expression of E-cadherin but suppressed the expression of snail and slug in A431 cells. It suggested that lapatinib can inhibit EMT, reducing drug resistance and suppressing CSCC progression. Moreover, the activation of ErK1/2 signaling pathway is reported to be correlation with EMT progress in cSCC. We observed that lapatinib inactivates ErK1/2 
signaling pathways with a marked inhibition of the phosphorylation of ErK1/2 in A431 cell lines, which lapatinib may contribute to the inhibitory effect of lapatinib on EMT. Therefore, lapatinib may act as a putative EMT-targeting agent that will be clinically helpful to reduce the morbidity and mortality of cSCC.

In conclusion, we have observed the anticancer effect of lapatinib and investigated the potential molecular mechanism of its cancer cell-killing effect in human cSCC A431 cell lines. The mechanisms of action of lapatinib were mainly ascribed to cell cycle arrest, autophagy induction, and EMT inhibition, and their related signaling pathways. lapatinib induced the inhibition of PI3K/Akt/mTOR and ErK1/2 signaling pathways, which contributes to autophagy-inducing and EMT-suppressing effects in A431 cell lines. Collectively, lapatinib may represent a promising anticancer drug that can be used for cSCC treatment. More studies are warranted to reveal other potential targets and potential mechanisms of lapatinib in the treatment of human cSCC.

\section{Acknowledgements}

We thank Qing Li (Cancer Center, Daping Hospital and Research Institute of Surgery, Third Military Medical University) for his free assistance of language editing. We thank all the people and patients who participated in this study.

\section{Competing Interests}

The authors have declared that no competing interest exists.

\section{References}

1. Stratigos A, Garbe C, Lebbe C, Malvehy J, del Marmol V, Pehamberger H, Peris K, Becker JC, Zalaudek I, Saiag P et al: Diagnosis and treatment of invasive squamous cell carcinoma of the skin: European consensus-based interdisciplinary guideline. European journal of cancer (Oxford, England : 1990) 2015, 51(14):1989-2007

2. Diepgen TL, Mahler V: The epidemiology of skin cancer. The British journal of dermatology 2002, 146 Suppl 61:1-6.

3. Karia PS, Han J, Schmults CD: Cutaneous squamous cell carcinoma: estimated incidence of disease, nodal metastasis, and deaths from disease in the United States, 2012. Journal of the American Academy of Dermatology 2013, 68(6):957-966.

4. Butler GJ, Neale R, Green AC, Pandeya N, Whiteman DC: Nonsteroidal anti-inflammatory drugs and the risk of actinic keratoses and squamous cell cancers of the skin. Journal of the American Academy of Dermatology 2005, 53(6):966-972

5. Muranushi C, Olsen CM, Pandeya N, Green AC: Aspirin and nonsteroidal anti-inflammatory drugs can prevent cutaneous squamous cell carcinoma: a systematic review and meta-analysis. The Journal of investigative dermatology 2015, 135(4):975-983

6. Higa GM, Abraham J: Lapatinib in the treatment of breast cancer. Expert review of anticancer therapy 2007, 7(9):1183-1192.

7. Burris HA, 3rd: Dual kinase inhibition in the treatment of breast cancer: initial experience with the EGFR/ErbB-2 inhibitor lapatinib. The oncologist 2004, 9 Suppl 3:10-15.

8. Jost M, Kari C, Rodeck U: The EGF receptor - an essential regulator of multiple epidermal functions. European journal of dermatology : EJD 2000, 10(7):505-510.

9. Wan YS, Wang ZQ, Shao Y, Voorhees JJ, Fisher GJ: Ultraviolet irradiation activates PI 3-kinase/AKT survival pathway via EGF receptors in human skin in vivo. International journal of oncology 2001, 18(3):461-466.

10. Barnes CJ, Bagheri-Yarmand R, Mandal M, Yang Z, Clayman GL, Hong WK, Kumar R: Suppression of epidermal growth factor receptor, mitogen-activated protein kinase, and Pak1 pathways and invasiveness of human cutaneous squamous cancer cells by the tyrosine kinase inhibitor ZD1839 (Iressa). Molecular cancer therapeutics 2003, 2(4):345-351.

11. Wei X, Duan W, Li Y, Zhang S, Xin X, Sun L, Gao M, Li Q, Wang D: AT101 exerts a synergetic efficacy in gastric cancer patients with 5-FU based treatment through promoting apoptosis and autophagy. Oncotarget 2016.

12. Wei X, Li Q, Li Y, Duan W, Huang C, Zheng X, Sun L, Luo J, Wang D, Zhang S et al: Prediction of survival prognosis of non-small cell lung cancer by APE1 through regulation of Epithelial-Mesenchymal Transition. Oncotarget 2016, 7(19):28523-28539.

13. Wei X, Li Q, Li Y, Duan W, Huang C, Zheng X, Sun L, Luo J, Wang D, Zhang S et al: Prediction of survival prognosis of non-small cell lung cancer by APE1 through regulation of Epithelial-Mesenchymal Transition. Oncotarget 2016.

14. Tomaskovic-Crook E, Thompson EW, Thiery JP: Epithelial to mesenchymal transition and breast cancer. Breast cancer research: BCR 2009, 11(6):213.

15. Song Y, Zhao C, Dong L, Fu M, Xue L, Huang Z, Tong T, Zhou Z, Chen A, Yang $Z$ et al: Overexpression of cyclin B1 in human esophageal squamous cell carcinoma cells induces tumor cell invasive growth and metastasis. Carcinogenesis 2008, 29(2):307-315

16. Lomas A, Leonardi-Bee J, Bath-Hextall F: A systematic review of worldwide incidence of nonmelanoma skin cancer. The British journal of dermatology 2012, 166(5):1069-1080.

17. Alam M, Ratner D: Cutaneous squamous-cell carcinoma. The New England journal of medicine 2001, 344(13):975-983.

18. Chu OS, Cianfrocca ME, Goldstein LJ, Gale M, Murray N, Loftiss I Arya N, Koch KM, Pandite L, Fleming RA et al: A phase I and pharmacokinetic study of lapatinib in combination with letrozole in patients with advanced cancer. Clinical cancer research : an official journal of the American Association for Cancer Research 2008, 14(14):4484-4490.

19. Chen ZL, Shen YW, Li ST, Li CL, Zhang LX, Yang J, Lv M, Lin YY, Wang X, Yang J: The efficiency and safety of trastuzumab and lapatinib added to neoadjuvant chemotherapy in Her2-positive breast cancer patients: a randomized meta-analysis. OncoTargets and therapy 2016, 9:3233-3247.

20. Liu CY, Hu MH, Hsu CJ, Huang CT, Wang DS, Tsai WC, Chen YT, Lee CH, Chu PY, Hsu CC et al: Lapatinib inhibits CIP2A/PP2A/p-Akt signaling and induces apoptosis in triple negative breast cancer cells. Oncotarget 2016, 7(8):9135-9149.

21. Cavallaro U, Christofori G: Cell adhesion and signalling by cadherins and Ig-CAMs in cancer. Nature reviews Cancer 2004, 4(2):118-132. 